\title{
ADENOMASTECTOMY WITH IMMEDIATE RECONSTRUCTION FOR THE TREATMENT OF INVASIVE TUMORS: COMPLICATIONS AND ONCOLOGIC SAFETY
}

\author{
Antonio Luiz Frasson¹, Martina Lichtenfels², Alessandra Borba Anton de Souza', Isabela Miranda, Fernanda \\ Barbosa $^{3}$ \\ ${ }^{1}$ Hospital São Lucas da PUCRS - Porto Alegre (RS), Brazil. \\ ${ }^{2}$ Latin American Cooperative Oncology Group - Porto Alegre (RS), Brazil. \\ ${ }^{3}$ Hospital Israelita Albert Einstein - Porto Alegre (RS), Brazil.
}

Introduction: Adenomastectomy, also called nipple-sparing mastectomy (NSM), consists of the surgical excision of all mammary gland tissue, with preservation of the skin and the nipple-areolar complex (NAC). In the past few years, different authors have been showing oncologic safety with the use of NSM, with similar recurrence and survival rates when compared to the total mastectomy technique. Besides, patients submitted to NSM present better aesthetic results and quality of life. This study aims at assessing the postoperative complications and local recurrence rates of patients with invasive breast cancer submitted to NSM with immediate reconstruction. Methods: This retrospective study collected data from patients with invasive tumors, submitted to NSM with immediate reconstruction, performed by the authors between 2001 and 2017 in Hospital São Lucas, at PUCRS (HSL), Hospital Moinhos de Vento (HMV), and Hospital Israelita Albert Einstein (HIAE). Results: a total of 198 patients diagnosed with invasive breast tumors were submitted to 374 NSM. Bilateral surgeries were performed in 176 patients, being 7 (4\%) patients diagnosed with bilateral invasive tumors; 8 (4.5\%), with ductal carcinoma in situ (DCIS) in the contralateral breast; $158.5 \%$ ) with atypia and/or lobular carcinoma in situ (LCIS); and 146 (83\%) patients who underwent risk-reducing surgery in the contralateral breast. Mean age of the patients was 45.3 years. Sentinel lymph node biopsy (SLNB) was performed in 143 (72.2\%) patients, and 25 (17.5\%) presented with positive lymph nodes. Twenty (10.1\%) patients were submitted to SLNB, followed by axillary lymph node dissection, and 35 (17.7\%) underwent axillary lymph node dissection. Assessing the molecular subtypes, 65 (32.9\%) tumors were luminal A; 71 (35.8\%), luminal B; 20 (10.1\%), luminal B/positive Her2; 15 (7.6\%), positive Her2; and 27 (13.6\%), triple negative. In the 374 performed NS, it was possible to observe $25(6.6 \%)$ postoperative complications, 10 (2.6\%) infections, $2(0.5 \%)$ hematomas, $5(1.3 \%)$ partial necrosis of the NAC, $8(2.2 \%)$ cases of dehiscence. After a mean follow-up time of 50 months, of the 198 patients who underwent NSM to treat cancer, 11 (5.5\%) presented local recurrence, being only $3(1.5 \%)$ recurrences in the NAC. Conclusions: After assessing all NSMs with immediate reconstruction carried out for the treatment of invasive cancer, only $6.6 \%$ presented postoperative complications. The rates of partial necrosis of the NAC (1.3\%) and infection (2.6\%) were low in comparison to findings from other authors. Besides, the local recurrence rates of $5.5 \%$ and $1.5 \%$ in NAC in patients submitted to NSM with immediate reconstruction for the treatment of invasive breast cancer are in accordance with the previous literature. These results show the oncologic safety of this procedure as a surgical therapeutic choice for the selected patients, demonstrating even lower rates of partial NAC necrosis. 